\title{
EXTREME QUASARS: OBSERVATIONS AND CONSTRAINTS
}

\author{
MARTIN ELVIS \\ Harvard-Smithsonian Center for Astrophysics, Cambridge MA 02138 USA
}

\begin{abstract}
The range of continuum shape the quasar phenomenon covers is larger than is usually thought, and is limited by observational sensitivity. between $1 / 3$ and $1 / 2$ of all quasars are "extreme" in at least one band. We have embarked on a program of observations to explore extreme spectral shapes, and to push to high redshift/luminosity. This paper presents X-ray and multi-wavelength SEDs for $12 \mathrm{z} \sim 3$ quasars.
\end{abstract}

\section{Extreme Quasars}

Over the past decade Spectral Energy Distributions (SEDs) have been compiled from the radio to X-ray bands for several dozen AGN and quasars (Malkan and Sargent 1982, Elvis et al. 1986, Neugebauer et al. 1987, Sanders et al. 1989, Elvis et al. 1994). All of the quasars with SEDs however are 'moderate', i.e. they have middling values of luminosity, low redshifts and are quite flat in their SEDs. This moderation is the result of the sensitivity of the IRAS, IUE and Einstein missions. Because each of these satellites had a limiting sensitivity only about a factor 10 below the flux of the brightest sources only sources with comparable $\nu f \nu$ across all three bands could have SEDs constructed. Any SED that strongly deviated from a flat distribution would not be detected. Thus the instruments define a 'Spectral Window Function' through which all observed SEDs must pass (Brissenden 1989, Elvis and Brissenden 1994). Even so, the observed SEDs show as much variation as is allowed by the constraints of the Spectral Window Function, about a factor 1020 (see McDowell, these proceedings). Other considerations (Elvis and Brissenden 1994) also suggest that the Spectral Window Function is limiting our understanding of the true range of quasar SEDs, so that perhaps $1 / 3-1 / 2$ of all quasars are extreme in at least one band.

With the advent of ROSAT, ASCA, HST and improved infrared detectors this window is being opened wider. We have taken the opportunity to search for ' $\mathrm{Ex}$ treme Quasars' and build SEDs for them. Our hope is that extreme conditions will highlight the basic dependencies of the many observed quasar properties, and so lead us to the physics. Mathur (these proceedings) presents SEDs for an ' $\mathrm{X}$-ray quiet quasar', and a 'Red Quasar'. Here we present SEDs of high redshift quasars.

\section{Physical Evolution Of Quasars}

Quasars evolve strongly with redshift such that the break point, $L^{*}$, of their luminosity function shifts to higher luminosities by a factor of 40-50 between $z=0.1$ and $\mathrm{z}=2$ (Boyle et al 1987) The physical interpretation of this population evolution for individual quasars is not clear. There are two basic options: long-lived and shortlived. In the long-lived case quasars are intrinsically rare and their present day 
remnants are massive. Individual quasars are born at high luminosities at $\mathrm{z} \sim 2-3$ and decline in luminosity gradually so that at the present epoch they emit at no more than 1-2\% of $\mathrm{L}_{E d d}$. As the quasar black holes grow larger, and become more starved of accreting matter, the continuum they produce is likely to change in form. In the opposite, short-lived, case quasars are common (so that most large galaxies had one at one time), but each is active only for a short time $\left(\sim 10^{8} \mathrm{yr}\right)$. In order not to require too much mass these quasars must be accreting close to their Eddington limits while they are active. In this case all quasar spectra are likely to be similar. While these expectations are qualitative, because there is no detailed physical evolution model of quasars at present, they do indicate how high $\mathrm{z}$ quasar SEDs could constrain and guide such modeling efforts.

In the past few years a dozen or so bright $(\mathrm{m}<18)$ high redshift $(\mathrm{z}>2.8)$ quasars have been discovered that are prime targets for SED construction.

\section{Quasar X-Ray Spectra at $z=3$}

We observed a sample of $14 \mathrm{z}=3$ quasars with ROSAT (detecting 12), radio, thermal infrared (where $10 \mu \mathrm{m}$ ground-based bolometers can go almost 10 times fainter than typical IRAS observations), near infrared (where new arrays can give spectra in the rest frame optical band), and optical (rest frame ultraviolet) (Elvis et al. 1994, Bechtold et al. 1994). ROSAT observes from $0.2-2.4 \mathrm{keV}$, and so covers the $\sim 1-10 \mathrm{keV}$ emitted band covered at low redshifts by Ginga (Williams et al. 1992). These quasars also match the Ginga quasars in evolved luminosity, at $10^{46}-10^{47} \mathrm{erg}$ $\mathrm{s}^{-1}$ in the emitted $2-10 \mathrm{keV}$ band (assuming isotropic emission), c.f. $10^{45} \mathrm{erg} \mathrm{s}^{-1}$ for Ginga.

Figures 1a,b show X-ray spectral slope against luminosity and redshift. The radio-quiet slopes must be treated with caution because of the small number of counts $(\sim 25)$. The mean spectral index of the radio-quiet quasars (if $\mathrm{N}_{H} \equiv \mathrm{N}_{H}(\mathrm{Gal})$ ) is $1.1 \pm 0.04$, and that of radio-loud quasars is $0.7 \pm 0.08$. We note: (1) The low redshift slope difference between radio-loud and radio-quiet quasars (Wilkes and Elvis 1987, Williams et al. 1992) persists at $\mathrm{z} \sim 3$, radio-quiet quasars having steeper slopes; (2) neither radio-quiet $(\Delta \bar{\alpha}<0.7,99 \%$ confidence) nor radio-loud $(\Delta \bar{\alpha}<0.3)$ quasars show any strong change in $2-10 \mathrm{keV}$ spectral slope with redshift, or with luminosity. The absence of any change in spectral index at early epochs when $L^{*}$ is $\sim 40 L^{*}(z=0)$ argues in favor of short lifetime quasar models. However, if radio-quiet quasars are intrinsically absorbed (see below) then they may evolve by $\Delta \bar{\alpha}=0.85 \pm 0.25$. Determining their absorption is thus crucial.

\subsection{X-Ray Absorption toward High z Quasars}

The ROSAT spectra of high $\mathrm{z}$ quasars showed unexpected strong absorption. For three out of four radio-loud quasars showed X-ray absorption at a level of $\sim 10^{22}$ atoms $\mathrm{cm}^{-2}$ ( $\mathrm{z}=\mathrm{z}$ (quasar), Elvis et al. 1994) so excess absorption is common, but not universal, at $z=3$. The absorber redshifts are unknown- they may be internal 

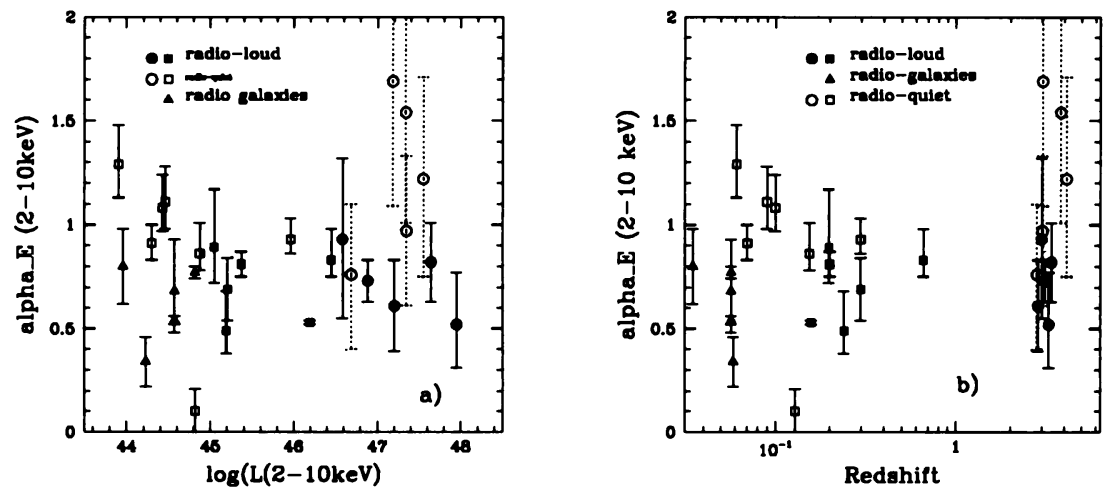

HO $=$ 50; OmegaO $=0$

Fig. 1. X-ray spectral slope $\left(f_{\nu} \propto \nu^{-\alpha}, 2-10 \mathrm{keV}\right)$ vs. (a) luminosity, (b) redshift.

(perhaps related to the Gigahertz Peaked nature of some of these radio sources), or intervening (possibly 'Damped Lyman- $\alpha$ ' systems at $\mathrm{z}<2$ ). The hardness ratios of the radio-quiet quasars suggest no strong absorption and so argues, weakly, for an intrinsic origin for the absorption in radio-loud quasars, since intervening absorption would be as likely for radio-quiet and radio-loud quasars. We refer the interested reader to Elvis et al (1994) for details.

\section{Quasar IR to UV SEDs at $z=3$}

Combining optical, near and thermal infrared we can produce SEDs for $z=3$ quasars such as the one shown in figure 2 (Kuhn et al 1994). Clearly this (and other) $\mathrm{z}=3$ quasars look similar to low $\mathrm{z}$ quasars, again favoring short-lived models (Bechtold et al 1994). The MMT $10 \mu \mathrm{m}$ bolometer point precludes any excess of $1000 \mathrm{~K}$ dust. Large amounts of hot dust are expected in some models (e.g. Haehnalt and Rees, 1993, these proceedings). Radio-quiet high $\mathrm{z}$, high luminosity quasars are more X-ray quiet (i.e. have larger $\alpha_{O} X$ ) than those at low $z$ and/or luminosity, but we cannot distinguish the two dependencies (Bechtold et al 1994). More subtle changes in the shape of the UV Bump may be present. The effects of reddening are now being investigated. Accretion disk models predict changes with $\mathrm{z}$ for long-lived quasars. First attempts to fit these models (Bechtold et al 1994) fail for large universes $\left(H_{0}=50, \Omega_{0}=0\right)$ in one case, but small universes $\left(H_{0}=100\right.$, $\Omega_{0}=1$ ) produce the observed UV slopes, and match the mass and accretion rate values derived solely from total luminosity requirements (Turner 1991).

\section{Conclusions}

It is now possible to gather SEDs for redshift 3 quasars across many bands in the electromagnetic spectrum. First indications are that no strong changes in the 


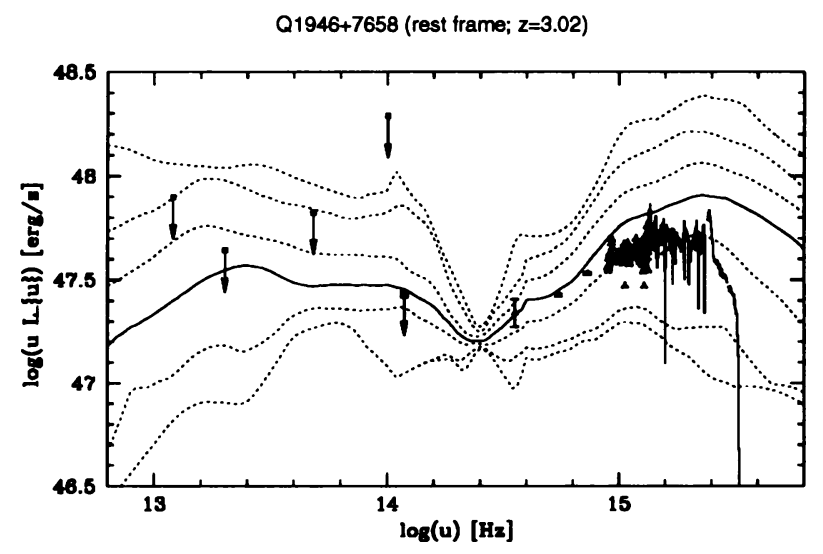

Fig. 2. IR to UV SED for the $z=3.02$ quasar HS1946 +7658 . The solid line is the mean SED for a low $z$ sample; the dashed lines are the $68 \% 90 \%$ and $99 \%$ ranges normalized at the near-IR inflection (see McDowell these proceedings).

continuum shape even while $L^{*}$ changes by a factor of $\sim 50$. This favors short-lived quasar models. More subtle effects may be present.

\section{Acknowledgements}

Jill Bechtold, Roc Cutri, Fabrizio Fiore, Olga Kuhn, Smita Mathur, Jonathan McDowell, Marcia Rieke, Aneta Siemiginowska, and Belinda Wilkes have all contributed to the present investigation. This work was supported in part by NASA grants NAGW-2201 (LTSARP), NAG5-1872 and NAG5-1536 (ROSAT), and NASA contracts NAS5-30934 (RSSDC), NAS5-30751 (HEAO-2) and NAS8-39073 (ASC).

\section{References}

Bechtold J., et al., 1994, Ap.J., submitted.

Boyle B., Fong R., and Shanks T., 1987, MNRAS, 227, 717.

Brissenden R.J.V., 1989, PhD Thesis, Australian National University.

Elvis M. et al., 1994, Ap.J.Suppl., submitted.

Elvis M. and Brissenden R.J.V. 1994, Ap.J., in preparation

Elvis M., et al., 1986, Ap.J., 310, 291.

Haehnalt M.G. and Rees M.J., 1993, MNRAS, 263, 168.

Kuhn O., Bechtold J., Cutri R., and Elvis M., 1994, Ap.J.Letters, submitted.

Neugebauer G., et al., 1987, Ap.J. Suppl., 63, 615.

Malkan M.A., and Sargent W.L.W., 1982, Ap.J., 254, 22.

Sanders D., et al 1989, Ap.J., 347, 29.

Turner E.L., 1991, A.J., 101, 5.

Wilkes B.J. and Elvis M., 1987, Ap.J., 323, 243.

Williams O.R., et al, 1992, Ap.J., 389, 157. 